\title{
Minimum Spanning Tree with Rough Weights
}

\author{
Sagarika Biswal \\ Department. of Mathematics \\ College of Engineering and Technology. \\ Bhubaneswar-751003, India
}

\author{
S. P. Mohanty \\ Department.of Mathematics \\ College of Engineering and Technology. \\ Bhubaneswar-751003, India
}

\begin{abstract}
In many real world problems related to weighted graphs, the input data corresponding to the weights are often imprecise due to incomplete or non-obtainable information. Finding the minimum spanning tree of such type of connected graphs is a challenge. This paper is introduced to find minimum spanning tree on a connected graph where the edges have rough weights.
\end{abstract}

\section{General Terms}

Graph Theory

\section{Keywords}

Rough set, rough variable, connected graph, spanning tree.

\section{INTRODUCTION}

The graph theory is effectively used to tackle may optimization problems. The classical graph theory is not well sufficient to deal with the problems where the data related to the graphs such as weight attached to the vertices, edges, the nature of connectivity (attributes) between the vertices are imprecise due to incomplete information. The fuzzy set theory has been used to deal with these type of problems where fuzzy weights are attached to the weighted graphs and different ranking methods are used to rank these fuzzy numbers to deal with the impreciseness. Fuzzy set theory is also ineffective if proper information regarding the nature of connectivity between the vertices is lacking. T.He et al [1],[2] have developed rough graph where they have applied rough set theory to attributed graph. Liang et al [3] has proposed edge rough graph to overcome some deficiencies of rough graph as proposed by T. He. S.P.Mohanty et al [4] have used the concept of T. He to find the minimum spanning tree (MST) of a rough graph where fuzzy numbers are attached as weights to the edges and the idea was explained through one numerical example to find the possibly conflicting MST.

A minimum spanning tree is a weighted connected graph, where the sum of the weights is minimal. In a graph $G=(V$, $\mathrm{E})$, where $\mathrm{V}$ is the set of vertices and $\mathrm{E}$ is the set of edges connecting the pair if vertices. There is one weight function $\mathrm{W}$ is defined which determines the weight of each edge. Creating a MST means to search for a subgraph $G^{1}=\left(V, E^{1}\right)$ of $\mathrm{G}$, where $\mathrm{E}^{\text {! }}$ is a subset of $\mathrm{E}$ and the total weights attached to the edges is minimum. Many algorithms are available in the literature to find MST which has wide application in many fields of Engineering and Computer Science. The famous Kruskal algorithm and Prim's algorithm are used to find MST for undirected graphs where the weights attached to the edges are precise. When the weights are not precise and there is uncertainty, many researchers have used fuzzy techniques to find MST.
In this paper, a method has been proposed to find the minimum spanning tree of a connected graph where rough weights are attached to the edges. Considering the $\alpha$ pessimistic value and $\alpha$-optimistic values of the rough weights, two such minimum spanning trees are obtained. Further two approaches are suggested for a compromise solution as regard to obtain one MST

The rest of the work is organized as follows: some properties of rough set, rough variables are given in section 2 . The problem formulation and solution method is given in section3. Two approaches along with the required algorithms are given in section 4 for getting a compromise solution. We conclude the work in section 5 with a suggestion for future work. Finally, in section 6 , we have presented one numerical example to implement the algorithm given in section 4 .

\section{PRILIMINARIES 2.1 Rough Set}

Rough set theory proposed by Z. Pawlak [5] is a mathematical tool for dealing with uncertain and incomplete data. Without any prior knowledge about the data, we deal only with the information provided by data to generate a conclusion. Since then, many researchers have developed the theoretical aspects and applied the concepts to solve problems related to diversified areas of science and engineering. Some fundamental definitions of rough set theory based on $\mathrm{Z}$. Pawlak are given below.

Let $\mathrm{U}$ be a finite, nonempty set called universe and let $\mathrm{R}$ be a binary relation on $\mathrm{U}$. Let $\mathrm{R}$ be an equivalence relation and $\mathrm{R}(\mathrm{x})=[\mathrm{x}]_{\mathrm{R}}$ be the equivalence class of the relation which contains $\mathrm{x}$. $\mathrm{R}$ shall be referred as indiscernibility relation.

For any $\mathrm{X} \subseteq \mathrm{U}$, the lower and upper approximation are defined as

$$
\begin{aligned}
& \underline{R}(\mathrm{X})=\{\mathrm{x} \in \mathrm{U}: \mathrm{R}(\mathrm{x}) \subseteq \mathrm{X}\} \\
& \bar{R}(\mathrm{X})=\{\mathrm{x} \in \mathrm{U}: \mathrm{R}(\mathrm{x}) \cap \mathrm{X} \neq \phi\} .
\end{aligned}
$$

The lower approximation $\underline{R}(\mathrm{X})$ is exact set containing $\mathrm{X}$ so that the objects in $\underline{R}(\mathrm{X})$ are members of $\mathrm{X}$ with certainty on the basis of knowledge in $\mathrm{R}$, while the objects in $\bar{R}(\mathrm{X})$ can be classified as possible members of $\mathrm{X}$.

The difference between the upper of the lower approximation will be called as the R-boundary of $\mathrm{X}$, and defined as 
$\mathrm{BN}_{\mathrm{R}}(\mathrm{X})=\bar{R}(\mathrm{X})-\underline{R}(\mathrm{X})$.

The set $\mathrm{X}$ is $\mathrm{R}$-exact iff $\mathrm{BN}_{\mathrm{R}}(\mathrm{X})=\phi$, otherwise the set is $\mathrm{R}$ rough.

\subsubsection{Membership function}

The membership function of rough set is defined by $\mu_{X}^{R}(x)=\frac{\operatorname{card}(X \cap R(x))}{\operatorname{card} R(x)}$

so that $\mu_{X}^{R}(x) \in[0,1]$

The membership function can be considered as a coefficient which expresses uncertainty of an element $x$ being an element of $\mathrm{X}$. The membership function can be useful to define the lower and upper approximation.

$$
\begin{aligned}
& \underline{R}(\mathrm{X})=\left\{\mathrm{x} \in \mathrm{U}: \mu_{X}^{R}(\mathrm{x})=1\right\} \\
& \bar{R}(\mathrm{X})=\left\{\mathrm{x} \in \mathrm{U}: \mu_{X}^{R}(\mathrm{x})>0\right\} \\
& \mathrm{BN}_{\mathrm{R}}(\mathrm{x})=\left\{\mathrm{x} \in \mathrm{U} ; 0<\mu_{X}^{R}(\mathrm{x})<1\right\}
\end{aligned}
$$

Various aspect of the rough set concept can be explained either by lower and upper approximation or by rough membership function.

\subsubsection{Rough variable}

The concepts of rough variable and uncertain programming are introduced by Liu. B [6].[7],[8]. Kundu et al [9] have applied the concept of rough variable in proposing a model for solid transportation problem.

The following definitions are based on Liu. B [6]

Definition 2.1: Let $\Lambda$ be a nonempty set, A be a $\sigma$-algebra of subset of $\Lambda, \Delta$ be an element of $\mathrm{A}$ and $\pi$ be a non-negative, real valued, additive set function on $\mathrm{A}$. Then $(\Lambda, \Delta, A, \pi)$ is called a rough space.

Definition 2.2: A rough variable $\xi$ on the rough space $(\Lambda, \Delta, A, \pi)$ is a measurable function from $\Lambda$ to the set of real numbers $\mathbf{R}$ such that for every Borel set $\mathrm{B}$ of $\mathrm{R}$, $\{\lambda \in \pi \mid \xi(\lambda) \in B\} \in A$

Then the lower and upper approximation of the rough variable is defined as

$$
\begin{aligned}
& \underline{\xi}=\{\xi(\lambda) \mid \lambda \in \Delta\} \\
& \bar{\xi}=\{\xi(\lambda) \mid \lambda \in \Lambda\}
\end{aligned}
$$

Definition 2.3: Let $(\Lambda, \Delta, A, \pi)$ be a rough space. Then the upper and lower trust of an event $\mathrm{A}$ is defined as

$$
\begin{aligned}
& \operatorname{Tr}(A)=\frac{\pi\{A\}}{\pi\{\Lambda\}} \\
& \operatorname{Tr}(A)=\frac{\pi\{A \cap \Delta\}}{\pi\{\Lambda\}}
\end{aligned}
$$

And the trust of the event $\mathrm{A}$ is defined as by

$$
\operatorname{Tr}(A)=\frac{1}{2}\{\operatorname{Tr}\{A\}+\operatorname{Tr}\{A\}\}
$$

The trust measure satisfies the followings

$$
\begin{aligned}
& \operatorname{Tr}(\Lambda)=1 \\
& \operatorname{Tr}(\phi)=0 \\
& \operatorname{Tr}(A) \leq \operatorname{Tr}(B), \text { where } A \subseteq B \\
& \operatorname{Tr}(A)+\operatorname{Tr}\left(A^{c}\right)=1
\end{aligned}
$$

Definition 2.4: Let $\xi_{1}, \xi_{2}$ be rough variables defined on the rough space $(\Lambda, \Delta, A, \pi)$. Then their sum and product are defined as

$$
\begin{aligned}
& \left(\xi_{1}+\xi_{2}\right)(\lambda)=\xi_{1}(\lambda)+\xi_{2}(\lambda) \\
& \left(\xi_{1} \cdot \xi_{2}\right)(\lambda)=\xi_{1}(\lambda) \cdot \xi_{2}(\lambda)
\end{aligned}
$$

Definition 2.5: Let $\xi$ be a rough variable on the rough space $(\Lambda, \Delta, A, \pi)$ and $\alpha \in(0,1]$ then

$\xi_{\text {sup }}(\alpha)=\sup \{r \mid \operatorname{Tr}\{\xi \geq r\} \geq \alpha\}$ is called $\alpha$-optimize value of $\xi$.

$\xi_{\text {inf }}(\alpha)=\inf \{r \mid \operatorname{Tr}\{\xi \leq r\} \geq \alpha\}$ is called $\alpha$-pessimistic value to $\xi$.

Definition 2.6: Let $\xi$ be a rough variable on the rough space $(\Lambda, \Delta, A, \pi)$. The expected value of $\xi$ is defined by $\mathrm{E}(\xi)=\int_{0}^{\infty} \operatorname{Tr}(\xi \geq r) d r-\int_{-\infty}^{0} \operatorname{Tr}(\xi \leq r)$.

Definition.2.7: The trust density function $\mathrm{f}: \mathrm{R} \rightarrow[0, \infty]$ of a rough variable $\xi$ is a function such that $\phi(x)=\int_{-\infty}^{x} f(y) d y$ holds for all $\mathrm{x} \in(-\infty, \infty)$, where $\phi$ is the trust distribution of $\xi$. If $\xi=([a, b],[c, d])$ be a rough variable such that $\mathrm{c} \leq \mathrm{a}<\mathrm{b} \leq \mathrm{d}$, then the trust distribution $\phi(x)=\operatorname{Tr}(\xi \leq x)$ is 


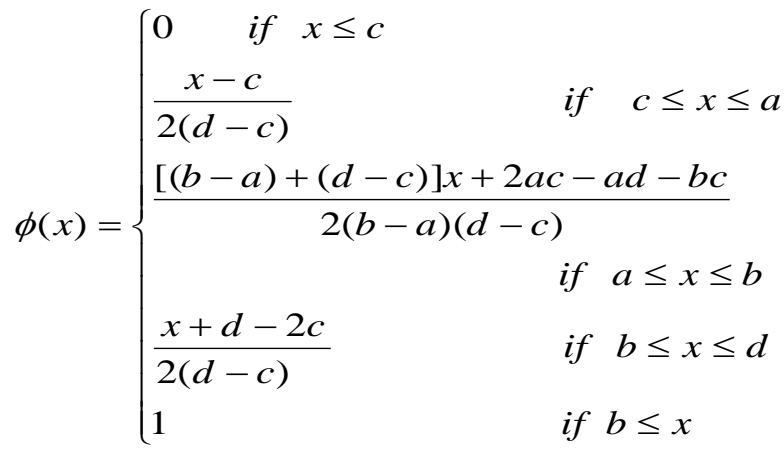

and the trust density function is defined as

$$
f(x)= \begin{cases}\frac{1}{2(d-c)} \quad \text { if } c \leq x \leq b \quad \text { or } b \leq x \leq d \\ \frac{1}{2(b-c)}+\frac{1}{2(d-c)} & \text { if } a \leq x \leq b \\ 0 \quad & \text { otherwise }\end{cases}
$$

$\alpha$-optimistic value to $\xi=([a, b],[c, d])$ is

$$
\xi_{\text {sup }}(\alpha)=\left\{\begin{array}{lc}
(1-2 \alpha) d+2 \alpha c, & \text { if } \alpha \leq \frac{d-b}{2(d-c)} \\
2(1-\alpha) d+(2 \alpha-1) c, & \text { if } \alpha \geq \frac{2 d-a-c}{2(d-c)} \\
\frac{d(b-a)+b(d-c)-2 \alpha(b-a)(d-c)}{(b-a)+(d-c)}, \text { otherwise }
\end{array}\right\}
$$

$\alpha$-pessimistic value of $\xi$ is

$$
\xi_{\text {inf }}(\alpha)=\left\{\begin{array}{l}
(1-2 \alpha) c+2 \alpha d, \quad \text { if } \alpha \leq \frac{a-c}{2(d-c)} \\
2(1-\alpha) c+(2 \alpha-1) d, \quad \text { if } \alpha \geq \frac{b+d-2 c}{2(d-c)} \\
\frac{c(b-a)+a(d-c)+2 \alpha(b-a)(d-c)}{(b-a)+(d-c)}, \text { otherwise }
\end{array}\right\}
$$

The expected value of $\xi$ is $\mathrm{E}(\xi)=\frac{1}{4}(a+b+c+d)$

\section{DESCRIPTION OF THE PROBLEM}

Let $\mathrm{G}=(\mathrm{V}, \mathrm{E})$ be a given connected and undirected graph. A spanning tree of $\mathrm{G}$ is a subset of edges $\mathrm{T} \subseteq \mathrm{E}$ such that $|\mathrm{T}|=$ $|\mathrm{V}|-1$ and the sub-graph $\mathrm{G}^{\prime}=(\mathrm{V}, \mathrm{T})$ is acyclic. Hence, the spanning tree of a graph is the minimal subset of edges that spans all vertices of $G$. The set of all spanning tree in $G$ is denoted by $\Gamma$. In the deterministic case, for every edge e $\in \mathrm{E}$, a real cost $c_{e}$ is attached and we wish to compute a minimum spanning tree $T$ of $G$, such that $T \in \Gamma$ whose total cost

$\mathrm{f}(\mathrm{T})=\sum_{e \in E} c_{e}$ is minimum.
In this work, the cost attached to the edges, are not precise. Due to uncertainty or unavailable information about the precise cost, each edge is assigned rough cost

$\mathrm{C}=([\mathrm{a}, \mathrm{b}],[\mathrm{c}, \mathrm{d}])$.

Initially, the value of $\alpha \in[0,1]$ is specified as trust level.

Let $\mathrm{W}_{\mathrm{i}, \mathrm{j}}=\left(\left[\mathrm{a}_{\mathrm{i}, \mathrm{j}}, \mathrm{b}_{\mathrm{i}, \mathrm{j}}\right],\left[\mathrm{c}_{\mathrm{i}, \mathrm{j}}, \mathrm{d}_{\mathrm{i}, \mathrm{j}}\right]\right.$ be the cost attached to the edge connecting the vertices $\mathrm{v}_{\mathrm{i}}, \mathrm{v}_{\mathrm{j}}$.

Let $W_{i j}^{1}$ be the $\alpha$-optimistic value of $\mathrm{W}_{\mathrm{i}, \mathrm{j}}$ and $W_{i j}^{11}$ be the $\alpha$ pessimistic value of $\mathrm{W}_{\mathrm{i}, \mathrm{j}}$. Hence,

$W_{i j}^{1}=\left\{\begin{array}{cc}(1-2 \alpha) c_{i j}+2 \alpha d_{i j} & \text { if } \alpha \leq \frac{a_{i j}-c_{i j}}{2\left(d_{i j}-c_{i j}\right)} \\ 2(1-\alpha) c_{i j}+(2 \alpha-1) d_{i j} & \text { if } \alpha \geq \frac{b_{i j}+d_{i j}-2 c_{i j}}{2\left(d_{i j}-c_{i j}\right)} \\ \frac{c_{i j}\left(b_{i j}-a_{i j}\right)+a_{i j}\left(d_{i j}-c_{i j}\right)+2 \alpha\left(b_{i j}-a_{i j}\right)\left(d_{i j}-c_{i j}\right)}{\left(b_{i j}-a_{i j}\right)+\left(d_{i j}-c_{i j}\right)} & \text { otherwise }\end{array}\right.$

$W_{i j}^{11}=\left\{\begin{array}{lc}(1-2 \alpha) d_{i j}+2 \alpha c_{i j} & \text { if } \alpha \leq \frac{d_{i j}-b_{i j}}{2\left(d_{i j}-c_{i j}\right)} \\ 2(1-\alpha) c_{i j}+(2 \alpha-1) d_{i j} & \text { if } \alpha \geq \frac{2 d_{i j}-a_{i j}-c_{i j}}{2\left(d_{i j}-c_{i j}\right)} \\ \frac{d_{i j}\left(b_{i j}-a_{i j}\right)+b_{i j}\left(d_{i j}-c_{i j}\right)-2 \alpha\left(b_{i j}-a_{i j}\right)\left(d_{i j}-c_{i j}\right)}{\left(b_{i j}-a_{i j}\right)+\left(d_{i j}-c_{i j}\right)} & \text { otherwise }\end{array}\right.$

Hence, two set of weights are generated basing on the $\alpha$ optimistic and $\alpha$-pessimistic values of the corresponding rough cost.

Considering each type of weights, the minimum spanning tree of the graph can be obtained using any standard algorithm.

\section{COMPROMISE SOLUTION}

In the process, we may get two different minimum spanning tree corresponding to each of $\alpha$-pessimistic value and $\alpha$ optimistic value. Choosing MST for $\alpha$-pessimistic value $\left(\mathrm{T}_{\mathrm{p}}\right)$ may increase the total optimistic value. Similarly, choosing the MST for $\alpha$-optimistic value $\left(\mathrm{T}_{\mathrm{o}}\right)$, may increase the total pessimistic value. The decision maker always prefers one MST having different $\alpha$-pessimistic value and $\alpha$-optimistic value for a easy decision. So it is ideal to find one MST with a compromise for increasing the $\alpha$-pessimistic value and reducing the $\alpha$-optimistic value or increasing the $\alpha$-optimistic value and reducing the $\alpha$-pessimistic value when we compare with the two such MST obtained corresponding to $\alpha$ pessimistic values or $\alpha$-optimistic values.

Two such approaches are underlined below for finding a compromise solution. 


\subsection{Preferential approach}

In this approach the edges connecting the nodes are prioritised by defining a norm $\mu$ on the edges.

Let $e_{i j}$ be the edge connecting the vertices $v_{i}$ and $v_{j}$. Let $w_{i j}{ }^{(p)}$ be the $\alpha$-pessimistic weight of $\mathrm{e}_{\mathrm{ij}}$ and $\mathrm{w}_{\mathrm{ij}}{ }^{(\mathrm{o})}$ be the $\alpha$-optimistic weight of $\mathrm{e}_{\mathrm{ij}}$. The norm $\mu$ is defined as $\mu=\left\|\mathrm{w}_{\mathrm{ij}}{ }^{\left({ }^{(0)}\right.}-\mathrm{w}_{\mathrm{ij}}{ }^{(\mathrm{p})}\right\|$ which is the absolute difference between two quantities.

The edge having less norm is preferred to be included in the spanning tree. This is appropriate in the sense that, more closeness in two values gives impreciseness less.

Considering the preferential edges the MST may be constructed by the following algorithm

\section{Algorithm: Preferential MST}

1. Let $\mathrm{G}=(\mathrm{V}, \mathrm{E})$ be the graph where $|\mathrm{V}|=\mathrm{n}$ and $|\mathrm{E}|=\mathrm{m}$

2. Choose a value for $\alpha$ as trust level

3. Find $\alpha$-pessimistic value $\mathrm{w}_{\mathrm{ij}}{ }^{(\mathrm{p})}$ and $\alpha$-optimistic value $\mathrm{w}_{\mathrm{ij}}{ }^{(\mathrm{o})}$ of each edge $\mathrm{e}_{\mathrm{ij}}$ connecting the vertices $\mathrm{v}_{\mathrm{i}}$ and $\mathrm{v}_{\mathrm{j}}$.

4. For $(I<=m)$ find the norm $\mu_{i}$ for the edge $e_{i}$

5. $\mathrm{T}=\Phi$

6. while $|\mathrm{T}|<\mathrm{n}-1$ do

Choose an edge e with minimal norm such that $\mathrm{T} U\{\mathrm{e}\}$ contains no cycle

7. end while

8. Find the weight of $\mathrm{T}$ corresponding to the $\alpha$-pessimistic value

9. Find the weight of $\mathrm{T}$ corresponding to the $\alpha$-optimistic value

10. Stop

\subsection{Edge-exchange between the MST}

Let $T_{p}$ be the MST obtained considering the $\alpha$-pessimistic value of the edges and $T_{0}$ be the MST obtained considering the $\alpha$-optimistic value of the edges. We can exchange two edges of the MST alternatively till we get both the MST coincide with a compromise $\alpha$-values.

\section{Algorithm: Exchange}

1. Let $\mathrm{G}=(\mathrm{V}, \mathrm{E})$ be the graph where $|\mathrm{V}|=\mathrm{n}$ and $|\mathrm{E}|=\mathrm{m}$

2. Choose a value for $\alpha$ as trust level

3. Find $\alpha$-pessimistic value $\mathrm{w}_{\mathrm{ij}}{ }^{(\mathrm{p})}$ and $\alpha$-optimistic value $\mathrm{w}_{\mathrm{ij}}{ }^{\left({ }^{(0)}\right.}$ of each edge $\mathrm{e}_{\mathrm{ij}}$ connecting the vertices $\mathrm{v}_{\mathrm{i}}$ and $\mathrm{v}_{\mathrm{j}}$.

4. Find $T_{p}$ and $T_{o}$, the MST corresponding to the $\alpha$ pessimistic values and $\alpha$-optimistic values respectively.

5. if $\left(\mathrm{T}_{\mathrm{p}} \neq \mathrm{T}_{\mathrm{o}}\right)$ then

\{

find an edge e in $T_{p}$ but not in $T_{o}$ and an edge $f$ in $T_{o}$ but not in $T_{p}$. Exchange the edge e in $T_{p}$ by $f$ if it does not generate a cycle\}

if $\left(T_{p} \neq T_{o}\right)$ then

find an edge $e$ in $T_{p}$ but not in $T_{o}$ and an edge $f$ in $T_{o}$ but not in $T_{p}$. Exchange the edge $f$ in $T_{o}$ by e if it does not generate a cycle\}
6. end if

7. if $\left(T_{p} \neq T_{o}\right)$ then goto step 5

8. end if

9. Find the weight of $T_{p}=T_{o}$ corresponding to the $\alpha$ pessimistic value

10. Find the weight of $T_{p}=T_{o}$ corresponding to the $\alpha$ optimistic value

11. Stop

\section{CONCLUSION}

Finding minimum spanning tree for a directed and undirected graph is a fundamental work in graph theory. Many efficient algorithms have been found out by many researchers to find MST in less time complexity. Algorithms and methods have been developed where the cost of edges is imprecise and fuzzy costs have been assigned to meet the impreciseness. Methods for bi-criteria MST have also been developed by many researchers to find MST under constraints. In our work, we have developed a technique to find MST for a undirected graph where the rough weights are attached to the edges. The work can be extended to the case of directed graph.

\section{NUMERICAL EXAMPLE}

Let us consider a graph with seven vertices and 11 edges, where rough weights are attached to each edge as per the Table 1. The trust level $\alpha$ is chosen at 0.9. The norm, $\alpha$ pessimistic and $\alpha$-optimistic values corresponding to the rough weights are given in the Table 2 . The graph is given in the Fig- 1 . The MST corresponding to the $\alpha$-pessimistic and $\alpha$ optimistic values are given in the Fig-2 and Fig-3. The MST for corresponding to the norms is given in the Fig-4 and finally a compromise MST taking the procedure of edge exchange in MST for $\alpha$-pessimistic values and MST for $\alpha$ optimistic values given in the Fig-5. In all cases the total minimum weights corresponding to $\alpha$-pessimistic, $\alpha$ optimistic values are given in the Table 3 .

Table 1. Rough weights attached to the edges

\begin{tabular}{|l|l|l|l|}
\hline $\mathrm{W}_{\mathrm{ab}}$ & $([1,3],[0.5,5])$ & $\mathrm{W}_{\mathrm{cg}}$ & $([8,9],[5,10])$ \\
\hline $\mathrm{W}_{\mathrm{af}}$ & $([6,7],[2,8]$ & $\mathrm{W}_{\mathrm{de}}$ & $([7,12],[6,15])$ \\
\hline $\mathrm{W}_{\mathrm{ag}}$ & $([2,6],[1,12]$ & $\mathrm{W}_{\mathrm{dg}}$ & $([5,9],[2,11])$ \\
\hline $\mathrm{W}_{\mathrm{bc}}$ & $([5,7],[2,10])$ & $\mathrm{W}_{\mathrm{ef}}$ & $([7,8],[2,10])$ \\
\hline $\mathrm{W}_{\mathrm{bg}}$ & $([6,10],[4,11])$ & $\mathrm{W}_{\mathrm{eg}}$ & $([6,9],[1,11])$ \\
\hline $\mathrm{W}_{\mathrm{cd}}$ & $([4,10],[5,11])$ & $\mathrm{W}_{\mathrm{fg}}$ & $([5,10],[4,12])$ \\
\hline
\end{tabular}


Table 2. $\alpha$-pessimistic, $\alpha$-optimistic and norm of edges

\begin{tabular}{|l|l|l|l|l|l|}
\hline $\mathrm{W}_{\mathrm{ab}}{ }^{1}$ & 4.1 & $\mathrm{~W}_{\mathrm{ab}}{ }^{11}$ & 1.1 & $\mu_{\mathrm{ab}}$ & 3 \\
\hline $\mathrm{W}_{\mathrm{af}}{ }^{1}$ & 6.97 & $\mathrm{~W}_{\mathrm{af}}{ }^{11}$ & 3.2 & $\mu_{\mathrm{af}}$ & 3.77 \\
\hline $\mathrm{W}_{\mathrm{ag}}{ }^{1}$ & 9.8 & $\mathrm{~W}_{\mathrm{ag}}{ }^{11}$ & 2.32 & $\mu_{\mathrm{ag}}$ & 7.58 \\
\hline $\mathrm{W}_{\mathrm{bc}}{ }^{1}$ & 8.4 & $\mathrm{~W}_{\mathrm{bc}}{ }^{11}$ & 3.6 & $\mu_{\mathrm{bc}}$ & 4.8 \\
\hline $\mathrm{W}_{\mathrm{bg}}{ }^{1}$ & 9.85 & $\mathrm{~W}_{\mathrm{bg}}{ }^{11}$ & 5.4 & $\mu_{\mathrm{bg}}$ & 4.45 \\
\hline $\mathrm{W}_{\mathrm{cd}}{ }^{1}$ & 9.9 & $\mathrm{~W}_{\mathrm{cd}}{ }^{11}$ & 5.1 & $\mu_{\mathrm{cd}}$ & 4.8 \\
\hline $\mathrm{W}_{\mathrm{cg}}{ }^{1}$ & 9.0 & $\mathrm{~W}_{\mathrm{cg}}{ }^{11}$ & 6.0 & $\mu_{\mathrm{cg}}$ & 3.0 \\
\hline $\mathrm{W}_{\mathrm{de}}{ }^{1}$ & 13.2 & $\mathrm{~W}_{\mathrm{de}}{ }^{11}$ & 7.29 & $\mu_{\mathrm{de}}$ & 5.91 \\
\hline $\mathrm{W}_{\mathrm{dg}}{ }^{1}$ & 9.2 & $\mathrm{~W}_{\mathrm{dg}}{ }^{11}$ & 3.8 & $\mu_{\mathrm{dg}}$ & 5.4 \\
\hline $\mathrm{W}_{\mathrm{ef}}{ }^{1}$ & 8.4 & $\mathrm{~W}_{\mathrm{ef}}{ }^{11}$ & 3.6 & $\mu_{\mathrm{ef}}$ & 4.8 \\
\hline $\mathrm{W}_{\mathrm{eg}}{ }^{1}$ & 9.0 & $\mathrm{~W}_{\mathrm{eg}}{ }^{11}$ & 3.0 & $\mu_{\mathrm{eg}}$ & 6.0 \\
\hline $\mathrm{W}_{\mathrm{fg}}{ }^{1}$ & 10.4 & $\mathrm{~W}_{\mathrm{fg}}{ }^{11}$ & 5.23 & $\mu_{\mathrm{fg}}$ & 5.23 \\
\hline
\end{tabular}

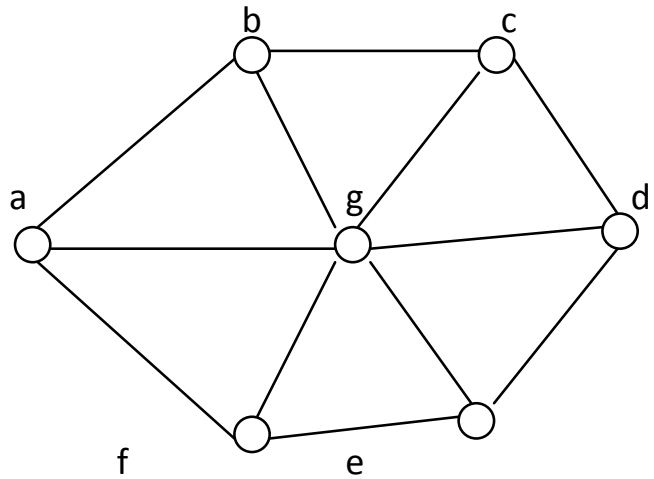

Fig 1: The original graph

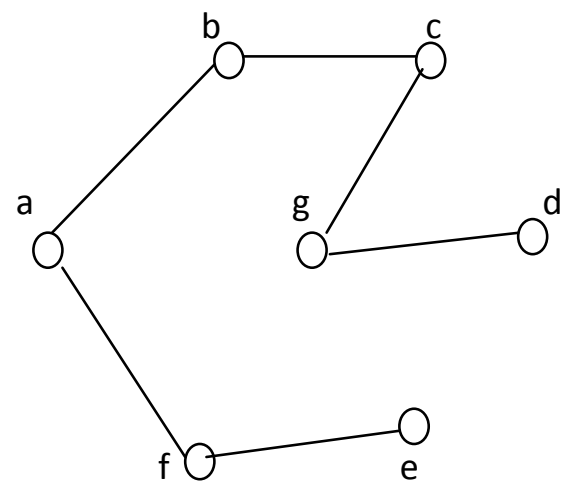

Fig 2: MST for $\alpha$-pessimistic value

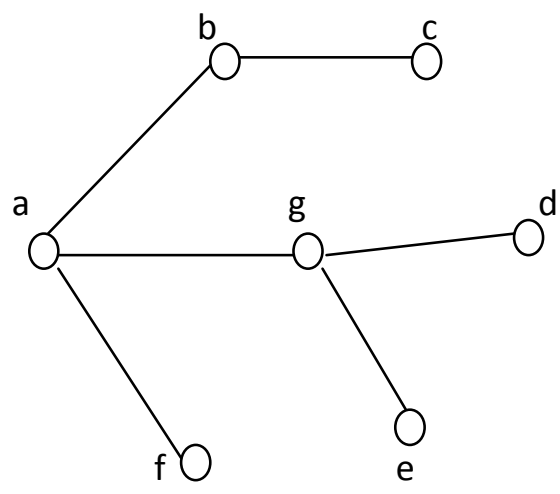

Fig 3: MST for $\alpha$-optimistic value

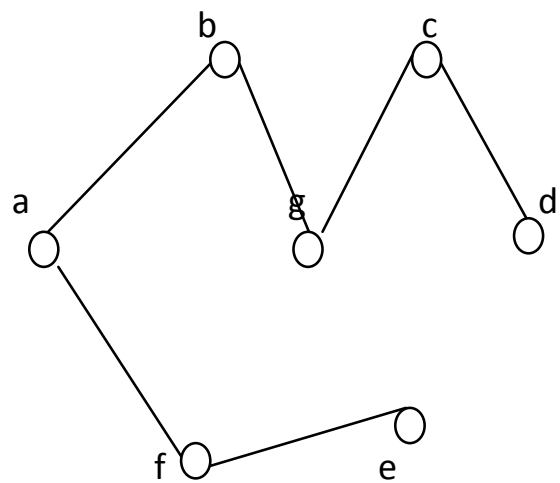

Fig 4: Preferential MST

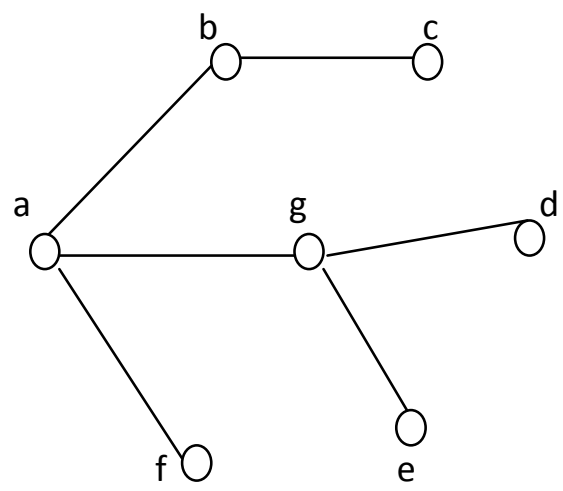

Fig 5: Edge exchange MST

Table 3: Comparison of different MST

\begin{tabular}{|l|l|l|}
\hline Type of MST & $\alpha$-optimistic value & $\begin{array}{l}\alpha \text {-pessimistic } \\
\text { value }\end{array}$ \\
\hline$\alpha$-pessimistic & 21.3 & 46.07 \\
\hline$\alpha$-optimistic & 17.02 & 47.27 \\
\hline Preferential & 24.4 & 48.22 \\
\hline Edge-exchange & 17.02 & 47.27 \\
\hline
\end{tabular}


In this example, the edge exchange MST and MST for $\alpha$ optimistic value coincides. This is due to the fact that only one pair of edges have been exchanged in MST for $\alpha$-pessimistic value. For larger graph, if the number of pairs is more, the edge exchange MST will be different which can be considered as a compromise solution.

\section{ACKNOWLEDGMENTS}

We offer our sincere thanks to the referee for his valuable suggestions for revision of the paper.

\section{REFERENCES}

[1] He, T, Chan, Y, Shi, K., "Weighted rough graphs and its application", Proc of sixth International Conference on Intelligent System Design and Application (ISDA 2006), 1, IEEE Computer Soc., 2006, 486-491

[2] He, T and Shi, K, " Rough graph and its structure", Journal of Shandong University, 6, 2006, 88-92
[3] Liang, M, Liang, B, Wei, L, Xu, X, "Edge rough graph and its application", Proc of Eighth Internaional Conference on Fuzzy Systems and Knowledge Discovery, 2011, 335-338

[4] Mohanty, S.P, Biswal, S, Pradhan,G, "Minimum spanning tree in fuzzy weighted rough graph", Int Journal of Engineering and Development", 10, 2012, 23 28

[5] Pawlak, Z, "Rough sets", Int Journal of Information and Computer Science", 11, 1982, 341-356

[6] Liu, B, "Uncertainty theory to its axiomatic foundation", 2004, Spinger-Verlag, Berlin.

[7] Liu, B, "Theory and practice of uncertain programming", 2002, Physical-Verlag, Heidelberg

[8] Liu, B, "Inequalities and convergence properties fuzzy and rough variables, "'Fuzzy Optimization and Decision Making, 2, 2003, 87-100

[9] Kundu, P, Kar, S, Maiti, M, "Some solid transportation models with crisp and rough costs", World Academy of Science, Engineering and Technology, 73, 2013, 185-192 